九州大学学術情報リポジトリ

Kyushu University Institutional Repository

\title{
AN \$ M TIMES N \$ CELL SPACE SURROUNDED BY TIME INVARIANT BOUNDARIES AND TWO INHIBITION STATES \$ PHI \$ : INFORMATION SCIENCE APPROACH TO BIOMATHEMATICS, XII
}

Kitagawa, Toshio

Research Institute of the Fundamental Information Science, Faculty of Science, Kyushu University

https://doi.org/10.5109/13064

出版情報: 統計数理研究. 15 (1/2)，pp. 67-84，1972-03. Research Association of Statistical Sciences バージョン :

権利関係 : 


\title{
AN $m \times n$ CELL SPACE SURROUNDED BY TIME INVARIANT BOUNDARIES AND TWO INHIBITION STATES $\phi$ \\ -INFORMATION SCIENCE APPROACH TO BIOMATHEMATICS, XII
}

By

\author{
Tosio KitagaWA*
}

(Received November 13, 1971)

\section{Introduction}

This paper gives some general observations on oscillatory phenomena associated with an $m \times n$ cell space surrounded by invariant boundary periphery plus two $\phi$ inhibition states. In fact in our previous paper [1], we discussed such oscillatory phenomenon for the simplest case when $m=n=2$, by means of direct enumeration of transitions among all configurations under application of local majority transformations LMT.

Although such an enumeration procedure would be too labourous and tedious to perform even for moderate values of $m$ and $n$, there are some fundamental aspects of oscillatory phenomena in our general formulation, which are both simple and easily obtained with reference to our LMT.

For the sake of the definiteness let us set up the following layout concerning our configurations as shown in Figure 1.1.

$$
\begin{array}{cccccccc}
\phi & d_{1} & d_{2} & d_{3} & \cdots & d_{j} & \cdots & d_{n} \gamma \\
a_{1} & x_{11} & x_{12} & x_{13} & \cdots & x_{1 j} & \cdots & x_{1 n} c_{1} \\
a_{2} & x_{21} & x_{22} & x_{23} & \cdots & x_{2 j} & \cdots & x_{2 n} c_{2} \\
a_{3} & x_{31} & x_{32} & x_{33} & \cdots & x_{3 j} & \cdots & x_{3 n} c_{3} \\
\vdots & \vdots & \vdots & \vdots & & \vdots & & \vdots \\
a_{i} & x_{i 1} & x_{i 2} & x_{i 3} & \cdots & x_{i j} & \cdots & x_{i n} c_{i} \\
\vdots & \vdots & \vdots & \vdots & & \vdots & & \vdots \\
a_{m} & x_{m 1} & x_{m 2} & x_{m 3} & \cdots & x_{m j} & \cdots & x_{m n} c_{m} \\
\alpha & b_{1} & b_{2} & b_{3} & \cdots & b_{j} & \cdots & b_{n}
\end{array}
$$

Fig. 1.1. $\phi^{2} \Omega_{m, n}\left(\left(x_{i j}\right) ; a, b, c, d ; \alpha, \gamma\right)$.

* Research Institute of the Fundamental Information Science, Faculty of Science, Kyushu University. 
An $m \times n$ cell space with configuration $\left(x_{i j}\right)$ surrounded by four time invariant vectors $a=\left(a_{1}, a_{2}, \cdots, a_{m}\right), b=\left(b_{1}, b_{2}, \cdots, b_{n}\right), c=\left(c_{1}, c_{2}, \cdots, c_{m}\right), d=\left(d_{1}, d_{2}, \cdots, d_{n}\right)$ and two time invariant corners $\alpha$ and $\gamma$ with two inhibition states $\phi$.

Definition 1.1. A rectangular set of $(m+2) \times(n+2)$ cells as shown in Figure 1.1 is called to be an $m \times n$ cell space, denoted by $\Omega_{m, n}$, surrounded by a set of four time invariant boundary vectors $a, b, c, d$ and two time invariant corner values $\alpha$ and $\gamma$ with the two $\phi$ inhibition states when the following conditions are satisfied:

$\left(1^{\circ}\right)$ The following four boundary vectors

$$
\begin{aligned}
& \text { (i) } \quad a=\left(a_{1}, a_{2}, \cdots, a_{i}, \cdots, a_{m}\right) \\
& \text { (ii) } c=\left(c_{1}, c_{2}, \cdots, c_{i}, \cdots, c_{m}\right) \\
& \text { (iii) } b=\left(b_{1}, b_{2}, \cdots, b_{j}, \cdots, b_{n}\right) \\
& \text { (iv) } d=\left(d_{1}, d_{2}, \cdots, d_{j}, \cdots, d_{n}\right)
\end{aligned}
$$

are time invariant under any application of LMT. Each of all the components of these four vectors is either 1 or 0.

$\left(2^{\circ}\right)$ The corners $\alpha$ and $\gamma$ are time invariant, having either one of 1 and 0.

$\left(3^{\circ}\right)$ Two $\phi^{\prime}$ s in the corner are inhibition state, which means a $2 \times 2$ basic cell space having $\phi$ element in one of cells is invariant under the application of LMT.

$\left(4^{\circ}\right)\left(x_{i, j}\right)(i=1,2, \cdots, m ; j=1,2, \cdots, n)$ in the cell space $\Omega_{m, n}$ denotes any arbitrarily given configuration in an $m \times n$ cell space.

The set of $(m+2) \times(n+2)$ cells defined by these conditions is denoted by

$$
\phi^{2} \Omega_{m, n}\left(\left(x_{i, j}\right) ; a, b, c, d ; \alpha, \gamma\right) .
$$

Particularly when we are concerned with the case when some of $a, b, c, d, \alpha$ and $\gamma$ are not specified, we shall denote each of unspecified elements by $*$. For instance

$$
\phi^{2} \Omega_{m, n}\left(\left(x_{i, j}\right) ; *, *, c, d ; *, \gamma\right)
$$

denotes the case when two boundary vectors $c$ and $d$ and one corner $\gamma$ are specified. but $a, b$ and $\alpha$ are unspecified, and similarly for

$$
\phi^{2} \Omega_{m, n}\left(\left(x_{i, j}\right) ; a, b, *, * ; \alpha, *\right) .
$$

In virtue of THEOREM 1 and its proof in Kitagawa and Yamaguchi [2], any specification of $\gamma, c$ and $d$ determines uniquely a stable configuration in $\Omega_{m, n}$ under the applications of LMT in which, in $\bmod 2$,

$$
x_{i, j}=\gamma+c_{i}+d_{j}=(\gamma c d)_{i, j}, \quad \text { say, for } i=1,2, \cdots, m ; j=1,2, \cdots, n .
$$

Similarly any specification of $\alpha, a$ and $b$ determines uniquely a stable configuration in $\Omega_{m, n}$ under the LMT in which, in $\bmod 2$,

$$
x_{i, j}=\alpha+a_{i}+b_{j}=(\alpha a b)_{i, j}, \quad \text { say, for } i=1,2, \cdots, m ; j=1,2, \cdots, n .
$$

Now if there were no interference with the boundary vectors $a, b$ and the corner $\alpha$, that is to say, all $a_{i}(i=1,2, \cdots, m)$ and $b_{j}(j=1,2, \cdots, n)$ and $\alpha$ reduce to be $\phi$, then we should reach to the configuration in the $(m+2) \times(n+2)$ cell space 


$$
\phi^{2} \Omega_{m, n}\left((\gamma c d) ; \phi_{a}, \phi_{b}, c, d ; \phi, \gamma\right)
$$

where we put

$$
\begin{array}{ll}
\phi_{a}=(\phi, \phi, \cdots, \phi), & (m \text {-dimensional }) \\
\phi_{b}=(\phi, \phi, \cdots, \phi), & (n \text {-dimensional }) .
\end{array}
$$

Similarly we could consider

$$
\phi^{2} \Omega_{m, n}\left((\alpha a b) ; a, b, \phi_{c}, \phi_{d} ; \alpha, \phi\right),
$$

where

$$
\begin{array}{ll}
\phi_{c}=(\phi, \phi, \cdots, \phi), & (m \text {-dimensional }) \\
\phi_{d}=(\phi, \phi, \cdots, \phi), & (n \text {-dimensional }) .
\end{array}
$$

The problems of configuration changes under application of LMT are concerned with the transition phenomena among configurations for each assigned set of configuration $C(X)=\left(x_{i j}\right)(i=1,2, \cdots, m ; j=1,2, \cdots, n), a, b, c, d, \alpha, \gamma$ and two $\phi$ states under applications of LMT, that is to say, with a set of configurations $C_{T}(X)$ induced in (1.02) by any application of LMT, which we shall denote by

$$
\begin{aligned}
& T \phi^{2} \Omega_{m, n}(C(X) ; a, b, c, d ; \alpha, \gamma) \\
= & \phi^{2} \Omega_{m, n}\left(C_{T}(X) ; a, b, c, d ; \alpha, \gamma\right) .
\end{aligned}
$$

In this paper we shall discuss these problems in a most general formulation. It is obvious that there are two types of transition phenomena of configuration $\left(x_{i, j}\right)$ under applications of LMT. The first type is concerned with transitory aspect of transition phenomena in which, starting with any assigned configuration in $\Omega_{m, n}$, we are seeking for a sequence of LMT's by which we can reach at a certain subset of all the configurations in $\Omega_{m, n}$ where we can never get out of, once we have reached any configuration belonging to tho set. We shall call such a set to be a final set of all the possible configurations in (1.04) under LMT, and which will be called to be the final set of configurations in the set of $a, b, c, d, \alpha$ and $\gamma$, which we shall denote by $\phi^{2} \Omega_{m, n}[a, b, c, d ; \alpha, \gamma]$.

Now the second type of our problems is concerned with an enumeration of all elements in $\phi^{2} \Omega_{m, n}[a, b, c, d ; \alpha, \gamma]$ each assigned set of $a, b, c, d, \alpha$ and $\gamma$, and with the transition phenomena among all the configurations in $\phi^{2} \Omega_{m, n}[a, b, c, d ; \alpha, \gamma]$. If it so happens that, for $i=1,2, \cdots, m ; j=1,2, \cdots, n$,

$$
(\alpha a b)_{i, j} \equiv(\gamma c d)_{i, j}, \quad(\bmod .2)
$$

then the final set of configurations in our concern consists of just one element, that is

$$
\begin{aligned}
\phi^{2} & \Omega_{m, n}[a, b, c, d ; \alpha, \gamma] \\
& =\left\{\phi^{2} \Omega_{m, n}\left((\alpha a b)_{i, j}\right) ; a, b, c, d ; \alpha, \gamma\right\} \\
& =\left\{\phi^{2} \Omega_{m, n}\left((\gamma c d)_{i, j}\right) ; a, b, c, d ; \alpha, \gamma\right\} .
\end{aligned}
$$


The so-called oscillatory phenomena, which we have already verified in the $\Omega_{m, n}$, occur when

$$
(\alpha a b)_{i, j} \equiv(\gamma c d)_{i, j}+1, \quad(\bmod .2)
$$

for all combinations of $i$ and $j$ in $i=1,2, \cdots, m ; j=1,2, \cdots, n$.

In what follows we shall proceed to a more complicated situation from a simpler one, step by step, with the start given in Section 2 .

2. The case of $\phi^{2} \Omega_{m, n}\left(C(X) ; 1_{a}, 1_{b}, 0_{c}, 0_{d} ; 1,0\right)$

In this Section we are entirely concerned with the case when, in (1.04), we have

$$
\begin{aligned}
& a=(1,1, \cdots, 1)=1_{a}, \\
& b=(1,1, \cdots, 1)=1_{b}, \\
& c=(0,0, \cdots, 0)=0_{c}, \\
& d=(0,0, \cdots, 0)=0_{d}, \\
& \alpha=1, \\
& \gamma=0,
\end{aligned}
$$

and hence with

$$
\phi^{2} \Omega_{m, n}\left(C(x) ; 1_{a}, 1_{b}, 0_{c}, 0_{d} ; 1,0\right) .
$$

Since we have, for $i=1,2, \cdots, m ; j=1,2, \cdots, n$,

$$
\begin{array}{ll}
(\alpha a b)_{i, j} \equiv 1+1+1 \equiv 1, & (\bmod .2) \\
(\gamma c d)_{i, j} \equiv 0+0+0 \equiv 0, & (\bmod .2) .
\end{array}
$$

The final set of configurations $\phi^{2} \Omega_{m, n}\left[1_{a}, 1_{b}, 0_{c}, 0_{d} ; 1,0\right]$ contains two configurations

$$
\phi^{2} \Omega_{m, n}\left(C_{0} ; 1_{a}, 1_{b}, 0_{c}, 0_{d} ; 1,0\right),
$$

and

$$
\dot{\phi}^{2} \Omega_{m, n}\left(C_{1} ; 1_{a}, 1_{b}, 0_{c}, 0_{d} ; 1,0\right),
$$

where the configuration in which all $x_{i, j}$ are equal to $0(1)$ is denoted by $C_{0}\left(C_{1}\right)$.

Now let us start with

LEMma 2.1. To any assigned configuration $C(X)$ in $\Omega_{m, n}$, there are a finite sequence of LMT denoted by $\left\{T_{i} ; i=1,2, \cdots, N_{0}\right\}$ such that

$$
\begin{gathered}
\phi^{2} \Omega_{m, n}\left(T_{N_{0}} T_{N_{0-1}} \cdots T_{2} T_{1} C(X) ; 1_{a}, 1_{b}, 0_{c}, 0_{d} ; 1,0\right) \\
=\phi^{2} \Omega_{m, n}\left(C_{0} ; 1_{a}, 1_{b}, 0_{c}, 0_{d} ; 1,0\right)
\end{gathered}
$$

and also a finite sequence of LMT, denoted by $\left\{T_{(j)} ; j=1,2, \cdots, N_{1}\right\}$ such that

$$
\begin{gathered}
\phi^{2} \Omega_{m, n}\left(T_{\left(N_{1}\right)} T_{\left(N_{1-1}\right)} \cdots T_{(2)} T_{(1)} C(X) ; 1_{a}, 1_{b}, 0_{c}, 0_{d} ; 1,0\right) \\
=\phi^{2} \Omega_{m, n}\left(C_{1} ; 1_{a}, 1_{b}, 0_{c}, 0_{d} ; 1,0\right),
\end{gathered}
$$


where the non-negative integers $N_{0}$ and $N_{1}$ are depending upon the configuration $C(X)$.

Before entering into the proof, let it be noted that, since in this Section we are exclusively concerned with the cell space $\phi^{2} \Omega_{m, n}\left(C(X) ; 1_{a}, 1_{b}, 0_{c}, 0_{d} ; 1,0\right)$, we may and we shall write

$$
\begin{gathered}
T_{N_{0}} T_{N_{0-1}} \cdots T_{2} T_{1} C(X)=C_{0} \\
T_{\left(N_{1}\right)} T_{\left(N_{1-1}\right)} \cdots T_{(2)} T_{(1)} C(X)=C_{1}
\end{gathered}
$$

in the place of (2.12) and (2.13) respectively.

Proof of LemMa 2.1. Let us beginn with the first row of $\Omega_{m, n}$. Let us denote by $I_{1}$ the set of all $j$ for which $x_{1, j}=1(1 \leqq j \leqq n)$, that is,

$$
I_{1}=\left\{j ; x_{1, j}=1,1 \leqq j \leqq n\right\} \text {. }
$$

If $I_{1}$ is a null set, then all is right with the first row, and we shall move to the second row. If $I_{1}$ is not a null set, then there is a positive integer $j_{1}$ which is the largest integer in the set $I_{1}$.

Now, since the cell $c_{1, j}$, has one upper adjacent cell belonging either to the boundary $d$ or to the corner $\gamma$, and also it has one right adjacent cell belonging either to the boundary $c_{1}$ or to the first row, it is always possible to find a $2 \times 2$ basic cell space in which the configuration is given by

$$
\left(\begin{array}{cc}
0 & 0 \\
x_{1, j_{1}} & 0
\end{array}\right)
$$

and to which an application of LMT reduces all the four elements of (2.17) into zero, while all the other elements in $\Omega_{m, n}$ remain invariant.

We can and we shall repeat such applications of LMT to the cell $c_{1, j}$ when $j$ belongs to the set $I_{1}$ according to the descending order of $j$ values, until we find that $I_{1}$ is reduced to a null set. Then we shall move to the second row of $\Omega_{m, n}$. Since all the elements in the first row have now zero, the similar argument can be applied to the second row, which leads us to the conclusion that there is a sequence of LMT by which all the elements of the second row in $\Omega_{m, n}$ are reduced to zero.

Proceeding in this way we shall find that there is a finite sequence $\left\{T_{h}\right\}(h=1$, $2, \cdots, N_{0}$ ) of LMT by which, starting with any assigned configuration $C(X)$ in $\Omega_{m, n}$, we obtain

$$
T_{N_{0}} T_{N_{0-1}} T_{N_{0-2}} \cdots T_{2} T_{1} C(X)=C_{0} .
$$

Similar argument holds true for the proof of an existence of a finite sequence of LMT $\left\{T_{(g)}\right\}\left(g=1,2, \cdots, N_{1}\right)$ enunciated in the second part of LEMMA 2.1, which will start with the considerations of the $m$-th row and the smallest integer $j_{m}$ for which $x_{m, p}=0$ in $1 \leqq p \leqq n$.

In view of this Lemma, we may start with $C_{0}$ as an initial configuration. A sequence of LMT with any assigned sequence of firing points yields us another configuration, which has a certain common feature among all the possible configurations of $m \times n$ cell space. To illustrate this, it is convenient to introduce 
Definition 2.1. A set $E$ of cells belonging to the cell space $\Omega_{m, n}$ is said to be left and lower inclusive, abbreviated by LLI, if, to each cell $c_{i, j}$ belonging to the set $E$, all the cells $\left\{c_{i \div p, j-q}\right\}(p=0,1,2, \cdots, m-i ; q=0,1,2, \cdots, j-1)$ belong to the set $E$.

Definition 2.2. A set $F$ of cells belonging to the cell space $\Omega_{m, n}$ is said to be right and upper inclusive, abbreviated by RUI, if, to each cell $c_{i, j}$ belonging to the set $F$, all the cells $\left\{c_{i-p, j+q}\right\}(p=0,1,2, \cdots, i-1 ; q=0,1,2, \cdots, n-j)$ belong to the set $F$.

We observe

COROLLARY 2.1. A set of cells $E$ is LLI if and only if the complementary set $\Omega_{m, n}-E$ is RUI.

EXAMPLE 2.1.

$$
\left(\begin{array}{llllllllll}
\phi & 0 & 0 & 0 & 0 & 0 & 0 & 0 & 0 & 0 \\
1 & 0 & 0 & 1 & 0 & 1 & 0 & 0 & 0 & 0 \\
1 & 0 & 1 & 0 & 0 & 0 & 0 & 0 & 0 & 0 \\
1 & 1 & 1 & 1 & 0 & 1 & 1 & 0 & 0 & 0 \\
1 & 1 & 0 & 0 & 1 & 0 & 0 & 0 & 0 & 0 \\
1 & 0 & 1 & 1 & 0 & 1 & 1 & 0 & 0 & 0 \\
1 & 1 & 1 & 1 & 1 & 1 & 1 & 1 & 1 & \phi
\end{array}\right)
$$

Fig. 3.2, (a). Non-LLI set and non-RUI set.

$$
\left(\begin{array}{llllllllll}
\phi & 0 & 0 & 0 & 0 & 0 & 0 & 0 & 0 & 0 \\
1 & 1 & 1 & 0 & 0 & 0 & 0 & 0 & 0 & 0 \\
1 & 1 & 1 & 1 & 0 & 0 & 0 & 0 & 0 & 0 \\
1 & 1 & 1 & 1 & 1 & 1 & 0 & 0 & 0 & 0 \\
1 & 1 & 1 & 1 & 1 & 1 & 0 & 0 & 0 & 0 \\
1 & 1 & 1 & 1 & 1 & 1 & 1 & 0 & 0 & 0 \\
1 & 1 & 1 & 1 & 1 & 1 & 1 & 1 & 1 & \phi
\end{array}\right)
$$

Fig. 3.2, (b). LLI set $E$ and $C[E]$ associated with $E$.

The set of all the LLI sets in $\Omega_{m, n}$, which will be denoted by $\mathfrak{L}_{m, n}$, constitutes a lattice, and similarly for the set of all the RUI set in $\Omega_{m, n}$ which will be denoted by $\Re_{m, n}$.

DEFINITION 2.3. For each set $E$ belonging to $\mathfrak{R}_{m, n}$, the configuration $C[E]$ associated with $E$ is defined by

$$
\begin{aligned}
x_{i, j} & =1 & & \text { if the cell } c_{i, j} \in E \\
& =0 & & \text { if the cell } c_{i, j} \in \Omega_{m, n}-E
\end{aligned}
$$

The situation which we have now presented can be illustrated by the following Example.

We observe

LEMMA 2.2. Any application of LMT in our cell space to any given configuratian $C[E]$ associated with a LLI set $E$, gives a configuration $C\left[E^{\prime}\right]$ associated with a LLI set $E^{\prime}$. Similarly for RUI.

PROOF. Any application of LMT gives a substantial change of configuration 
when in the corresponding $2 \times 2$ basic cell space we have

$$
\left.x_{i, j}+x_{i, j+1}+x_{i+1, j}+x_{i+1, j+1} \equiv 1 \quad \text { (mod. } 2\right) \text {. }
$$

In view of the assumption that $E$ is a LLI set, all the possible configurations in the basic $2 \times 2$ cell space are either of the following four configurations.

$$
\left(\begin{array}{ll}
0 & 0 \\
1 & 0
\end{array}\right), \quad\left(\begin{array}{ll}
0 & 1 \\
0 & 0
\end{array}\right), \quad\left(\begin{array}{ll}
1 & 0 \\
1 & 1
\end{array}\right), \quad\left(\begin{array}{ll}
1 & 1 \\
0 & 1
\end{array}\right),
$$

each of which is transformed to each of the following ones respectively

$$
\left(\begin{array}{ll}
0 & 0 \\
0 & 0
\end{array}\right), \quad\left(\begin{array}{ll}
0 & 0 \\
0 & 0
\end{array}\right), \quad\left(\begin{array}{ll}
1 & 1 \\
1 & 1
\end{array}\right), \quad\left(\begin{array}{ll}
1 & 1 \\
1 & 1
\end{array}\right)
$$

by the application of LMT. By each of these local changes in the basic cell, $C(E)$ is transformed to another configuration $C\left(E^{\prime}\right)$ where $E^{\prime}$ is again a LLI set. This completes the proof of the first part of LEMMA 2.2. Similarly for the second part by means of the notion of RUI sets, which completes the whole proof of LEMMA 2.2.

In view of these Lemmas and their proofs it is immediate to observe

LEMMA 2.3. ( $\left.1^{\circ}\right)$ The set of all LLI sets (and hence RUI sets) in $\Omega_{m, n}$ constitutes a lattice with the unity $\Omega_{m, n}$ and zero element $\Phi$ according to the semi-ordered relation included by the set inclusion relation.

$\left(2^{\circ}\right)$ To any pair of two configurations $C[E]$ and $C[F]$ associated with to LLI sets $E$ and $F$ respectively such that $E \subset F$ in the lattice defined in $\left(1^{\circ}\right)$, there is a finite sequence of LMT's $\left\{T_{i}\right\}\left(i=1,2, \cdots, N_{0}\right)$ such that

$$
\begin{array}{ll}
\text { (i) } & T_{i} T_{i-1} \cdots T_{2} T_{1} E=E_{i} \quad\left(i=1,2, \cdots, N_{0}\right) \\
\text { (ii) } & E_{N_{0}}=F \\
\text { (iii) } & E \subset E_{1} \subset E_{2} \cdots E_{N_{0-1}} \subset E_{N_{0}} .
\end{array}
$$

$\left(3^{\circ}\right)$ To any pair of two configurations $C[E]$ and $C[F]$ associated with two RUI sets $E$ and $F$ respectively such that $E \supset F$ in the lattice defined in $\left(1^{\circ}\right)$, there is a finite sequence of LMT's $\left\{T_{(j)}\right\}\left(j=1,2, \cdots, N_{1}\right)$ such that

$$
\begin{array}{ll}
\text { (i) } & T_{(j)} T_{(j-1)} \cdots T_{(2)} T_{(1)} E=E_{j} \quad\left(j=1,2, \cdots, N_{1}\right) \\
\text { (ii) } & E_{N_{1}}=F \\
\text { (iii) } & E \supset E_{1} \supset E_{2} \supset \cdots \supset E_{N_{1}-1} \supset E_{N_{1}} .
\end{array}
$$

Proof. Since $\left(1^{\circ}\right)$ is obvious and $\left(3^{\circ}\right)$ is similar to $\left(2^{\circ}\right)$, it will be sufficient to show $\left(2^{\circ}\right)$. Now let us denote by $k$ the number of cells in the difference $F-E$. The proof will be done by induction as to $k$. First let $k=1$. Let us denote the cell belonging to $F-E$ by $c_{i, j}$. Then the $2 \times 2$ basic cell space consisting of $c_{i, j-1}, c_{i, j}, c_{i+1, j-1}$ and $c_{i+1, j}$ has the configuration

$$
\left(\begin{array}{ll}
1 & 0 \\
1 & 1
\end{array}\right)
$$

In the consequence there is a LMT having the firing point in this $2 \times 2$ basic cell space which transforms (2.25) into 
and hence transforms the configuration $C[E]$ into $C[F]$.

Now let us assume that the assertion in $\left(2^{\circ}\right)$ is valid for $k=h$. Let us prove that it is valid for $k=h+1$. Since both $E$ and $F$ are LLI set, there is at least one cell $c_{p, q}$ belonging to $F-E$ such that the $2 \times 2$ basic cell space consisting of $c_{p, q-1}$, $c_{p, q}, c_{p+1, q-1}$ and $c_{p+1, q}$ has the configuration as in (2.24), because of the fact that each of these cells belongs either to the set $E$ or to the $a, b$ boundaries and the corner $\alpha$, whose value is equal to 1 . The application of LMT at this $2 \times 2$ basic cell space reduces the number $h+1$ of the set $F-E$ to the number $h$ of the transformed set for which the assertion holds true. This completes the induction and hence the proof of $\left(2^{\circ}\right)$.

It is convenient to introduce

DEFINITION 2.3. (a) The set of all LLI sets in $\Omega_{m, n}$ is called to be the LLI family and denoted by $(\mathrm{BR})_{m, n}$.

(b) The set of all RUI sets in $\Omega_{m, n}$ is called to be the RUI family and denoted by $(\mathfrak{R H})_{m, n}$.

In combination of the three Lemmas and Definitions in this Section, we are now able to make clear the whole transition features of configurations in our particular cell space. In fact we have

THEOREM 2.1. In a cell space $\phi^{2} \Omega_{m, n}\left(C(X) ; 1_{a}, 1_{b}, 0_{c}, 0_{d} ; 1,0\right)$ we have the following assertions:

$\left(1^{\circ}\right)$ (Transitory aspect) To each pair of any assigned configuration $C(X)$ and any assigned configuration $C[E](\overline{C[F]})$ which is associated with a LLI set $E$ (a RUI set $F)$ there exists a finite sequence of LMT $\left\{T_{i}\right\}\left(\left\{T_{(j)}\right\}\right)$ whose sequential applications in this order transforms $C(X)$ into $C[E](\overline{C[F}])$.

$\left(2^{\circ}\right)$ (The final set of configurations) $\phi^{2} \Omega_{m, n}\left(1_{a}, 1_{b}, 0_{c}, 0_{d} ; 1,0\right)$ is equal to the set of all the configurations $C[E](\overline{C[F]})$ when $E$ runs through (R\&) $)_{m, n}$ (F runs through $\left.(\Re \mathfrak{H})_{m, n}\right)$, that is,

$$
\begin{aligned}
\phi^{2} \Omega_{m, n}\left(1_{a}, 1_{b}, 0_{c}, 0_{d} ; 1,0\right) & =\left[C[E] ; E \in(\mathfrak{Q R})_{m, n}\right] \\
& =\left[C[F] ; F \in(\mathfrak{H H})_{m, n}\right] .
\end{aligned}
$$

$\left(3^{\circ}\right)$ Once a configuration $C(X)$ coincides with a configuration $C[E](\overline{C[F]}$ corresponding to a LLI set $E$ (an RUI set $F$ ), then any application of LMT can only give another configuration $C\left[E^{\prime}\right]\left(\overline{C\left[F^{\prime}\right]}\right)$ corresponding to a LLI set $E^{\prime}$ (a RUI set $\left.F^{\prime}\right)$.

\section{3. $\phi^{2} \Omega_{m, n}$ cell space with conjugate configuration boundaries and corners}

Let us consider a cell space $\phi^{2} \Omega_{m, n}((\alpha a b) ; a, b, *, * ; \alpha, *)$, in which the configuration $\left(x_{i, j}\right)$ in $\Omega_{m, n}$ is given by

$$
x_{i, j}=\alpha+a_{i}+b_{j}, \quad(\bmod .2)(i=1,2, \cdots, m ; j=1,2, \cdots, n),
$$

with an assigned triple of $\alpha, a$ and $b$, while the boundary vectors $c, d$ and the cor- 
ner $\gamma$ are not specified, and also let us consider a cell space $\phi^{2} \Omega_{m, n}((\gamma c d) ; *, *, c, d$; $0, \gamma)$ in which the configuration $\left(y_{i, j}\right)$ in $\Omega_{m, n}$ is given by

$$
y_{i, j}=\gamma+c_{i}+d_{j}, \quad(\bmod .2),(i=1,2, \cdots, m ; j=1,2, \cdots, n)
$$

in a similar way. In this Section we are interested with the case when there hold

$$
x_{i, j}+1 \equiv y_{i, j}, \quad(\bmod .2),
$$

for all $i$ and $j$ in $\Omega_{m, n}$.

For this purpose let us introduce a set of conjugate configuration boundaries and corners in which there hold

$$
\begin{aligned}
& c_{i} \equiv a_{i}+1 \\
& d_{j} \equiv b_{j}+1 \quad(\bmod .2) \\
& \gamma \equiv \alpha+1
\end{aligned}
$$

for $i=1,2, \cdots, m$ and $j=1,2, \cdots, n$. That is to say, we are concerned with a cell space

$$
\phi^{2} \Omega_{m, n}(C(X) ; a, b, \bar{a}, \bar{b} ; \alpha, \bar{\alpha}),
$$

where bar notations mean the conjugate vector and conjugate value, and we are interested with the behaviour of configuration $C(X)$ in $\Omega_{m, n}$. There is no stable configuration in (3.07), as we can easily observe from the conditions (3.04)-(3.06). It is also immediate to observe

COROLlaRY 3.1. $\phi^{2} \Omega_{m, n}\left(C(X) ; 1_{a}, 1_{b}, 0_{c}, 0_{d} ; 1,0\right)$ has a set of conjugate configuration boundaries and corners.

It may be interesting to establish some results regarding oscillatory phenomena in our $\phi^{2} \Omega_{m, n}(C(X) ; a, b, \bar{a}, \bar{b} ; \alpha, \bar{\alpha})$ which can be recognized as generalizations of the results obtained in Section 2. However such an analogue is not so simple as one might imagine at its immediate conjecture. Let us explain the situation by the following

EXAMPLE 3.1. Let us consider the case when $m=8, n=9$ and

$$
\begin{aligned}
& a-(1,1,0,0,1,0,0,1) \\
& b=(1,0,0,0,1,1,0,0,1) \\
& c=(0,0,1,1,0,1,1,0)=\bar{a} \\
& d=(0,1,1,1,0,0,1,1,0)=\bar{b} \\
& \alpha=1, \gamma=0=\bar{\alpha} .
\end{aligned}
$$

The configuration $C[(\alpha a b)]$ is not stable at the upper and right corner as shown in Figure 3.1, (a) and the configuration $C[(\gamma c d)]$ is not stable at the lower and left corner as shown in Figure 3.1, (b). 


\begin{tabular}{l|lllllllll|l}
$\phi$ & 0 & 1 & 1 & 1 & 0 & 0 & 1 & 1 & 0 & 0 \\
\hline 1 & 1 & 0 & 0 & 0 & 1 & 1 & 0 & 0 & 1 & 0 \\
1 & 1 & 0 & 0 & 0 & 1 & 1 & 0 & 0 & 1 & 0 \\
0 & 0 & 1 & 1 & 1 & 0 & 0 & 1 & 1 & 0 & 1 \\
0 & 0 & 1 & 1 & 1 & 0 & 0 & 1 & 1 & 0 & 1 \\
1 & 1 & 0 & 0 & 0 & 1 & 1 & 0 & 0 & 1 & 0 \\
0 & 0 & 1 & 1 & 1 & 0 & 0 & 1 & 1 & 0 & 1 \\
0 & 0 & 1 & 1 & 1 & 0 & 0 & 1 & 1 & 0 & 1 \\
1 & 1 & 0 & 0 & 0 & 1 & 1 & 0 & 0 & 1 & 0 \\
\hline 1 & 1 & 0 & 0 & 0 & 1 & 1 & 0 & 0 & 1 & $\phi$
\end{tabular}

Fig. 3.1, (a). Configuration $C[(\alpha a b)]$.

\begin{tabular}{c|ccccccccc|c}
$\phi$ & 0 & 1 & 1 & 1 & 0 & 0 & 1 & 1 & 0 & 0 \\
\hline 1 & 0 & 1 & 1 & 1 & 0 & 0 & 1 & 1 & 0 & 0 \\
1 & 0 & 1 & 1 & 1 & 0 & 0 & 1 & 1 & 0 & 0 \\
0 & 1 & 0 & 0 & 0 & 1 & 1 & 0 & 0 & 1 & 1 \\
0 & 1 & 0 & 0 & 0 & 1 & 1 & 0 & 0 & 1 & 1 \\
1 & 0 & 1 & 1 & 1 & 0 & 0 & 1 & 1 & 0 & 0 \\
0 & 1 & 0 & 0 & 0 & 1 & 1 & 0 & 0 & 1 & 1 \\
0 & 1 & 0 & 0 & 0 & 1 & 1 & 0 & 0 & 1 & 1 \\
1 & 0 & 1 & 1 & 1 & 0 & 0 & 1 & 1 & 0 & 0 \\
\hline 1 & 1 & 0 & 0 & 0 & 1 & 1 & 0 & 0 & 1 & $\dot{\phi}$
\end{tabular}

Fig. 3.1, (b). Configuration $C[(\gamma c d)]$.

\begin{tabular}{c|c|c|c|c|cccccc|c}
$\phi$ & 0 & 1 & 1 & 1 & 0 & 0 & 1 & 1 & 0 & 0 \\
\hline 1 & 0 & 1 & 1 & 1 & 0 & 0 & 1 & 1 & 0 & 0 \\
\hline 1 & 1 & 1 & 1 & 1 & 0 & 0 & 1 & 1 & 0 & 0 \\
\hline 0 & 0 & 1 & 0 & 0 & 1 & 1 & 0 & 0 & 1 & 1 \\
\hline 0 & 0 & 1 & 1 & 1 & 0 & 0 & 1 & 0 & 1 & 1 \\
\hline 1 & 1 & 0 & 0 & 0 & 1 & 1 & 0 & 1 & 0 & 0 \\
\hline 0 & 0 & 1 & 1 & 1 & 0 & 0 & 1 & 0 & 1 & 1 \\
\hline 0 & 0 & 1 & 1 & 1 & 0 & 0 & 1 & 0 & 1 & 1 \\
\hline 1 & 1 & 0 & 0 & 0 & 1 & 1 & 0 & 0 & 0 & 0 \\
\hline 1 & 1 & 0 & 0 & 0 & 1 & 1 & 0 & 0 & 1 & $\phi$
\end{tabular}

Fig. 3.1, (c). $\phi^{2} \Omega_{8,9}\left(C\left(X_{1}\right) ; a, b, \bar{a}, \bar{b} ; 1,0\right)$ Mixed configuration of $(\alpha a b)$ and $(\gamma c d)$ acording to a LLI set $E$ shown by dotted boundary. 
Let us consider a LLI set $E$ (and hence RUI set $F=\Omega_{8,9}-E$ ) in which the configuration is coincident with $(\alpha a b)$ and elsewhere it is coincident with $(\gamma c d)$, which has a conjugate value of $(\alpha a b)$ in each corresponding cell. An example is shown in Figure 3.1, (c).

Now the difficulty comes from the specific property of local majority transformation LMT itself. By a formal application of LMT, we can not reach neither to $C(\alpha a b)$ nor to $C(\gamma c d)$ in $\Omega_{m, n}$, from the configuration given in Figure 3.1, (c), which we denote by $\phi^{2} \Omega_{8,9}\left(C\left(X_{1}\right) ; a, b, \bar{a}, \bar{b} ; 1,0\right)$, unless we would introduce a certain kind of interpretation about the roles of boundary vectors and corners. In fact it is important to observe that the time invariance of boundaries and corners should be interpreted to penetrate, in a certain sense, into the cell space $\Omega_{m, n}$.

Our interpretation of application of LMT to any assigned configuration (3.07) is based upon the following notions given by the following Definitions.

DEFINITION 3.1. To each assigned configuration $C(X)$ in (3.07), the difference configuration of $C(X)$ with respect to $C(\alpha a b)$ is defined by the configuration $\left(y_{i, j}\right)(i=1$, $2, \cdots, m ; j=1,2, \cdots, n)$ where

$$
y_{i, j} \equiv x_{i, j}-\left(\alpha+a_{i}+b_{j}\right) \quad(\bmod .2)
$$

and is denoted by

$$
C(X ; \alpha \beta \gamma)=C(X)-C(\alpha a b) .
$$

By virture of this Definition we now introduce

Definition 3.2. The (Boolean) sum of two $\phi^{2} \Omega_{m, n}$ cell spaces is defined by

$$
\begin{aligned}
& \sum_{i=1}^{2} \phi^{2} \Omega_{m, n}\left(C\left(X_{i}\right) ; a_{i}, b_{i}, c_{i}, d_{i} ; \alpha_{i}, \gamma_{i}\right) \\
& \quad=\phi^{2} \Omega_{m, n}\left(C\left(X_{1}+X_{2}\right) ; a_{1}+a_{2}, b_{1}+b_{2}, c_{1}+c_{2}, d_{1}+d_{2}, \alpha_{1}+\alpha_{2}, \gamma_{1}+\gamma_{2}\right),
\end{aligned}
$$

where we have put

$$
\begin{aligned}
C\left(X_{1}+X_{2}\right) & =C\left(X_{1}\right)+C\left(X_{2}\right) \\
& =\left(x_{i, j}^{(1)}\right)+\left(x_{i, j}^{(2)}\right) \\
& =\left(x_{i, j}^{(1)}+x_{i, j}^{(2)}\right)
\end{aligned}
$$

as usual matrix Boolean sum.

We note

COROLlaRy 3.2. Any $\phi^{2} \Omega_{m, n}$ cell space with the time invariant conjugate boundaries and corners with $\alpha=1$ can be considered to be the sum of two $\phi^{2} \Omega_{m, n}$ cell spaces in the following form

$$
\begin{aligned}
\phi^{2} \Omega_{m, n} & (C(X) ; a, b, \bar{a}, \bar{b} ; 1,0) \\
= & \phi^{2} \Omega_{m, n}(C[(\overline{\alpha a b})] ; \vec{a}, \bar{b}, \vec{a}, \bar{b} ; 0,0) \\
& \quad+\phi^{2} \Omega_{m, n}\left(C(X ; \overline{\alpha a b}) ; 1_{a}, 1_{b}, 0_{c}, 0_{d} ; 1,0\right) .
\end{aligned}
$$

This is immediate from the following relations: 
and

$$
\begin{aligned}
& a=\bar{a}+1_{a}, \quad b=\bar{b}+1_{b}, \\
& c=c+0_{c}=\bar{a}+0_{c}, \quad d=d+0_{d}=b+0, \\
& \alpha=1=\bar{\alpha}+1, \quad \bar{\alpha}=\bar{\alpha}+0,
\end{aligned}
$$

$$
\begin{aligned}
C[(\overline{\alpha a b})] & =C[(\bar{\alpha} \bar{a} \bar{b})] \\
& =\left(\bar{\alpha}+\bar{a}_{i}+\bar{b}_{j}\right) \\
& =\left((\alpha+1)+\left(a_{i}+1\right)+\left(b_{j}+1\right)\right) \\
& =\left(\alpha+a_{i}+b_{j}+1\right) \\
C(X ; \overline{\alpha a b}) & =\left(x_{i, j}-\left(\alpha+a_{i}+b_{j}+1\right)\right) .
\end{aligned}
$$

The interpretations of applications of LMT in $\phi^{2} \Omega_{m, n}$ cell space with the time invariant conjugate boundaries and corners is due to the following

AsSumption I. Any application of LMT in a cell space $\phi^{2} \Omega_{m, n}$ defined by the left hand side of (3.12) is equal to the sum of the results of application of LMT to each summand of the right-hand side of (3.12):

$$
\begin{aligned}
L \phi^{2} \Omega_{m, n}(C(X) ; a, b, \vec{a}, \bar{b} ; 1,0) & \\
= & L \phi^{2} \Omega_{m, n}(C[(\overline{\alpha a b})] ; \bar{a}, \vec{b}, \bar{a}, \bar{b} ; 0,0) \\
& \quad+L \phi^{2} \Omega_{m, n}\left(C(X ; \overline{\alpha a b}) ; 1_{a}, 1_{b}, 0_{c}, 0_{d} ; 1,0\right) .
\end{aligned}
$$

In view of the fact that

$$
\begin{aligned}
& L \phi^{2} \Omega_{m, n}(C[(\overline{\alpha a b})] ; \bar{a}, \bar{b}, \bar{a}, \bar{b} ; 0,0) \\
& \quad=\phi^{2} \Omega_{m, n}(C[(\overline{\alpha a b})] ; \bar{a}, \bar{b}, \bar{a}, \bar{b} ; 0,0)
\end{aligned}
$$

that is, stable in $\phi^{2} \Omega_{m, n}$, the whole problem is now reduced to the particular case discussed in Section 2.

\begin{tabular}{c|c|c|c|c|c|c|c|c|c|c}
$\phi$ & 0 & 1 & 1 & 1 & 0 & 0 & 1 & 1 & 0 & 0 \\
\hline 1 & 0 & 0 & 0 & 0 & 0 & 0 & 0 & 0 & 0 & 0 \\
\hline 1 & 1 & 1 & 0 & 0 & 0 & 0 & 0 & 0 & 0 & 0 \\
\hline 0 & 1 & 0 & 1 & 1 & 0 & 0 & 0 & 1 & 0 & 1 \\
\hline 0 & 1 & 0 & 0 & 0 & 1 & 1 & 0 & 0 & 0 & 1 \\
\hline 1 & 0 & 1 & 0 & 0 & 0 & 0 & 1 & 1 & 0 & 0 \\
\hline 0 & 0 & 0 & 1 & 0 & 0 & 1 & 0 & 1 & 0 & 1 \\
\hline 0 & 0 & 0 & 0 & 1 & 1 & 0 & 1 & 0 & 0 & 1 \\
\hline 1 & 0 & 0 & 0 & 0 & 0 & 1 & 0 & 0 & 0 & 0 \\
\hline 1 & 1 & 0 & 0 & 0 & 1 & 1 & 0 & 0 & 1 & $\phi$
\end{tabular}

Fig. 3.2. $\phi^{2} \Omega_{8,9}(C(X) ; a, b, \bar{a}, \bar{b} ; 1,0)$. 
EXAMPLE 3.2. Let us explain the decomposition given by (3.12) by the following two $\phi^{2} \Omega_{8,9}$ given in Figures 3.1 , (c) and 3.2 .

In the first place let us construct the cell space

$$
\phi^{2} \Omega_{8,9}(C(\overline{\alpha a b}) ; \bar{a}, \bar{b}, \bar{a}, \bar{b} ; 0,0),
$$

which is shown in Figure 2.4 .

\begin{tabular}{l|lllllllll|l}
$\phi$ & 0 & 1 & 1 & 1 & 0 & 0 & 1 & 1 & 0 & 0 \\
\hline 0 & 0 & 1 & 1 & 1 & 0 & 0 & 1 & 1 & 0 & 0 \\
0 & 0 & 1 & 1 & 1 & 0 & 0 & 1 & 1 & 0 & 0 \\
1 & 1 & 0 & 0 & 0 & 1 & 1 & 0 & 0 & 1 & 1 \\
1 & 1 & 0 & 0 & 0 & 1 & 1 & 0 & 0 & 1 & 1 \\
0 & 0 & 1 & 1 & 1 & 0 & 0 & 1 & 1 & 0 & 0 \\
1 & 1 & 0 & 0 & 0 & 1 & 1 & 0 & 0 & 1 & 1 \\
1 & 1 & 0 & 0 & 0 & 1 & 1 & 0 & 0 & 1 & 1 \\
0 & 0 & 1 & 1 & 1 & 0 & 0 & 1 & 1 & 0 & 0 \\
\hline 0 & 0 & 1 & 1 & 1 & 0 & 0 & 1 & 1 & 0 & $\phi$
\end{tabular}

Fig. 3.3. $\phi^{2} \Omega_{8,9}(C(\overline{\alpha a b}) ; \bar{a}, \bar{b}, \bar{a}, \bar{b}, 0,0)$.

Now we can obtain

$$
\begin{aligned}
\phi^{2} \Omega_{8,9}( & \left.C\left(X_{1}\right) ; a, b, \bar{a}, \bar{b} ; 1,0\right) \\
= & \phi^{2} \Omega_{8,9}(C[(\overline{\alpha a b})] ; \bar{a}, \bar{b}, \bar{a}, \bar{b} ; 0,0) \\
& +\phi^{2} \Omega_{8,9}\left(C\left(X_{1}, \overline{\alpha a b}\right) ; 1_{a}, 1_{b}, 0_{c}, 0_{d} ; 1,0\right),
\end{aligned}
$$

where the second summand in the right-hand side is shown in Figure 3.4.

\begin{tabular}{l|lllllllll|l}
$\phi$ & 0 & 0 & 0 & 0 & 0 & 0 & 0 & 0 & 0 & 0 \\
\hline 1 & 0 & 0 & 0 & 0 & 0 & 0 & 0 & 0 & 0 & 0 \\
1 & 1 & 0 & 0 & 0 & 0 & 0 & 0 & 0 & 0 & 0 \\
1 & 1 & 1 & 0 & 0 & 0 & 0 & 0 & 0 & 0 & 0 \\
1 & 1 & 1 & 1 & 1 & 1 & 1 & 1 & 0 & 0 & 0 \\
1 & 1 & 1 & 1 & 1 & 1 & 1 & 1 & 0 & 0 & 0 \\
1 & 1 & 1 & 1 & 1 & 1 & 1 & 1 & 0 & 0 & 0 \\
1 & 1 & 1 & 1 & 1 & 1 & 1 & 1 & 0 & 0 & 0 \\
1 & 1 & 1 & 1 & 1 & 1 & 1 & 1 & 1 & 0 & 0 \\
\hline 1 & 1 & 1 & 1 & 1 & 1 & 1 & 1 & 1 & 1 & $\phi$
\end{tabular}

Fig. 3.4. $\phi^{2} \Omega_{8,9}\left(C\left(X_{1}, \overline{\alpha a b}\right) ; 1_{a}, 1_{b}, 0_{c}, 0_{d} ; 1,0\right)$.

Similarly we have

$$
\begin{aligned}
\phi^{2} \Omega_{8,9}( & \left.C\left(X_{2}\right) ; a, b, \bar{a}, \bar{b} ; 1,0\right) \\
= & \phi^{2} \Omega_{8,9}(C[\overline{\alpha a b}] ; \bar{a}, \bar{b}, \bar{a}, \bar{b} ; 0,0) \\
& +\phi^{2} \Omega_{8,9}\left(C\left(X_{2} ; \overline{\alpha a b}\right) ; 1_{a}, 1_{b}, 0_{c}, 0_{d} ; 1,0\right),
\end{aligned}
$$


where the second summand in the right-hand side is shown in Figure 3.5.

\begin{tabular}{c|ccc|c|c|c|c|c|c|c}
$\phi$ & 0 & 0 & 0 & 0 & 0 & 0 & 0 & 0 & 0 & 0 \\
\hline 1 & 0 & 1 & 1 & 1 & 0 & 0 & 1 & 1 & 0 & 0 \\
\hline 1 & 1 & 0 & 1 & 1 & 0 & 0 & 1 & 1 & 0 & 0 \\
\hline 1 & 0 & 0 & 1 & 1 & 1 & 1 & 0 & 1 & 0 & 0 \\
\hline 1 & 0 & 0 & 0 & 0 & 0 & 0 & 0 & 0 & 1 & 0 \\
\hline 1 & 0 & 0 & 1 & 1 & 0 & 0 & 0 & 0 & 0 & 0 \\
\hline 1 & 1 & 0 & 1 & 0 & 1 & 0 & 0 & 1 & 1 & 0 \\
\hline 1 & 1 & 0 & 0 & 1 & 0 & 1 & 1 & 0 & 1 & 0 \\
\hline 1 & 0 & 0 & 1 & 1 & 0 & 1 & 1 & 1 & 1 & 0 \\
\hline 1 & 1 & 1 & 1 & 1 & 1 & 1 & 1 & 1 & 1 & $\phi$
\end{tabular}

Fig. 3.5. $\phi^{2} \Omega_{8,9}\left(C\left(X_{2} ; \overline{\alpha a b}\right) ; 1_{a}, 1_{b}, 0_{c}, 0_{d} ; 1,0\right)$.

After the consideration of implications of the time invariant properties of boundaries and corners as illustrated in EXAMPLE 3.1, it is convenient to introduce

Definition 3.3. A configuration $C(Z)$ in a cell space $\phi^{2} \Omega_{m, n}(C(Z) ; a, b, \bar{a}, \bar{b} ; 1,0)$ is said to be associated with a LLI set $E$ if $E$ is a LLI set in $\Omega_{m, n}$ and, for $i=1$, $2, \cdots, m$ and $j=1,2, \cdots, n$, we have

$$
z_{i, j}=\bar{\alpha}+\bar{a}_{i}+\bar{b}_{j}+e_{i, j},
$$

where

$$
e_{i, j}= \begin{cases}1 & \text { if the cell } c_{i, j} \in E \\ 0 & \text { if the cell } c_{i, j} \bar{E}\end{cases}
$$

that is to say, we have

$$
C(Z)=C(\overline{\alpha a b})+C[E]
$$

and

$$
\begin{aligned}
\phi^{2} \Omega_{m, n} & (C(Z) ; a, b, \bar{a}, \bar{b} ; 1,0) \\
= & \phi^{2} \Omega_{m, n}(C[\overline{\alpha a b}] ; \bar{a}, \bar{b}, \bar{a}, \bar{b} ; 0,0) \\
& +\phi^{2} \Omega_{m, n}\left(C[E] ; 1_{a}, 1_{b}, 0_{c}, 0_{d} ; 1,0\right) .
\end{aligned}
$$

We now enunciate

THEOREM 3.1. In the set of all the configurations in $\phi^{2} \Omega_{m, n}[a, b, \bar{a}, \bar{b} ; 1,0]$ for any assigned pair of $a$ and $b$, we have the following assertions:

$\left(1^{\circ}\right)$ (Transitory aspect) To each pair of any assigned configuration $C(X)$ in $\Omega_{m, n}$ and any assigned LLI set $E$, there exists a finite sequence of $\operatorname{LMT}\left\{T_{i}\right\}(i=1,2, \cdots$, $N_{0}$ ) such that the repeated applications of the sequence in this order give us 


$$
\begin{aligned}
& \left(\prod_{i=N_{0}}^{1} T_{i}\right) \phi^{2} \Omega_{m, n}(C(Z) ; a, b, \bar{a}, \bar{b} ; 1,0) \\
& \left.\quad=\phi^{2} \Omega_{m, n}(C \overline{\alpha a b}]+C[E] ; a, b, \bar{a}, \bar{b} ; 1,0\right)
\end{aligned}
$$

$\left(2^{\circ}\right)$ (Final aspect) $\phi^{2}\left(_{m, n}(\overline{\alpha a b})\right.$ is the final set of configurations in $\phi^{2} \Omega_{m, n}[(a, b, \bar{a}, \bar{b}$; $1,0)$ in the sense that, besides the assertion $\left(1^{\circ}\right)$, there holds the assertion that any application of LMT to any configuration belonging to $\phi^{2} \widetilde{S}_{m, n}(\overline{\alpha a b})$ can only give a configuration belonging to $\phi^{2}\left(\zeta_{m, n}(\overline{\alpha a b})\right.$.

$\left(3^{\circ}\right)$ (Oscillatory aspect) To each pair of any assigned two configurations belonging to $\phi^{2}\left(\S_{m, n}(\overline{\alpha a b})\right.$ there exists a finite sequence of LMT which transforms the one into the other.

THEOREM 3.1 is a generalization of THEOREM 2.1 which corresponds to the case when $a=1_{a}$ and $b=1_{b}$. The proof of THEOREM 3.1 is immediate from that of THEOREM 2.1. The enunciation of THEOREM 2.1 can be reduced to that similar to THEOREM 3.1.

\section{4. $\phi^{2} \Omega_{m, n}$ cell space under general boundaries}

We now proceed to consider a cell space $\phi^{2} \Omega_{m, n}(C(X) ; a, b, c, d ; 1,0)$ without any connection among $a, b, c$, and $d$. In view of the decomposition principle introduced in Section 4 , we have the decomposition

$$
\begin{aligned}
\phi^{2} \Omega_{m, n} & (C(X) ; a, b, c, d ; 1,0) \\
= & \phi^{2} \Omega_{m, n}((\overline{\alpha a b}) ; \bar{a}, \bar{b}, c, d ; 0,0) \\
& +\phi^{2} \Omega_{m, n}\left(C(X ; \overline{\alpha a b}) ; 1_{a}, 1_{b}, 0_{c}, 0_{d} ; 1,0\right) .
\end{aligned}
$$

Now it is noted that the first summand in the right-hand side of (4.01) is not stable in general, because we can not expect to have the relation $\bar{a}=c, \bar{b}=d$ under our general boundary configuration. In order to have a quick understanding of the behaviour induced by applications of LMT to the first summand of (4.01), it is convenient to observe an illustrative example.

EXAMPLE 4.1. Let us consider $\phi^{2} \Omega_{8,9}((\overline{\alpha a b}) ; \bar{a}, \bar{b}, c, d ; 0,0)$, where

$$
\begin{aligned}
& \bar{a}=(1,1,0,0,1,0,0,0) \\
& \bar{b}=(1,0,1,0,1,1,1,0,0) \\
& c=(1,1,1,0,0,0,0,1) \\
& d=(0,1,1,1,0,0,0,1,0) \\
& \alpha=0, \quad \gamma=0 .
\end{aligned}
$$

In Figure 4.1, (a), the configuration $C(\overline{\alpha a b})$ is given, which has so many unstable $2 \times 2$ basic cell spaces along the upper and along the right boundaries. In Figure 4.1 , (b), the configuration $C(\overline{\gamma c d})$ is given, which has now so many unstable $2 \times 2$ basic cell spaces along the lower and along the left boundaries. In $\Omega_{m, n}$ the values of some cells change while those of other cells remain unchanged in both of two 


\begin{tabular}{|c|c|c|c|c|c|c|c|c|c|c|}
\hline$\phi$ & 0 & 1 & 1 & 1 & 0 & 0 & 0 & 1 & 0 & 0 \\
\hline 1 & 0 & 1 & 0 & 1 & 0 & 0 & 0 & 1 & 1 & 0 \\
\hline 1 & 0 & 1 & $\theta$ & 1 & 0 & 0 & 0 & 1 & 1 & 1 \\
\hline 0 & 1 & 0 & 1 & $\theta$ & 1 & 1 & 1 & 0 & 0 & 1 \\
\hline 0 & 1 & 0 & 1 & 0 & 1 & 1 & 1 & 0 & 0 & 0 \\
\hline 1 & 0 & 1 & 0 & 1 & 0 & 0 & $\theta$ & 1 & 1 & 0 \\
\hline 0 & 1 & 0 & 1 & 0 & 1 & 1 & 1 & 0 & 0 & 0 \\
\hline 0 & 1 & 0 & 1 & 0 & 1 & 1 & 1 & 0 & $\theta$ & 0 \\
\hline 0 & 1 & 0 & 1 & 0 & 1 & 1 & 1 & 0 & 0 & 1 \\
\hline 0 & 1 & 0 & 1 & 0 & 1 & 1 & 1 & 0 & 0 & $\phi$ \\
\hline
\end{tabular}

Fig. 4.1, (a). $\phi^{2} \Omega_{8,9}((\overline{\alpha a b}) ; \bar{a}, \bar{b}, c, d ; 0,0)$.

\begin{tabular}{|c|c|c|c|c|c|c|c|c|c|c|}
\hline$\phi$ & 0 & 1 & 1 & 1 & 0 & 0 & 0 & 1 & 0 & 0 \\
\hline 1 & 1 & 0 & $\theta$ & 0 & 1 & 1 & 1 & 0 & 1 & 1 \\
\hline 1 & 1 & 0 & 0 & 0 & 1 & 1 & 1 & 0 & 1 & 1 \\
\hline 0 & 1 & 0 & 0 & $\theta$ & 1 & 1 & 1 & $\theta$ & 1 & 1 \\
\hline 0 & 0 & 1 & 1 & 1 & 0 & 0 & 0 & 1 & 0 & 0 \\
\hline 1 & 0 & 1 & 1 & 1 & 0 & 0 & 0 & 1 & 0 & 0 \\
\hline 0 & 0 & 1 & 1 & 1 & 0 & 0 & 0 & 1 & 0 & 0 \\
\hline 0 & 0 & 1 & 1 & 1 & 0 & 0 & 0 & 1 & 0 & 0 \\
\hline 0 & 1 & 0 & 0 & 0 & 1 & 1 & 1 & $\theta$ & 1 & 1 \\
\hline 0 & 1 & 0 & 1 & 0 & 1 & 1 & 1 & 0 & 0 & $\phi$ \\
\hline
\end{tabular}

Fig. 4.1, (b). $\phi^{2} \Omega_{8,9}((\overline{\gamma c d}) ; \bar{a}, \bar{b}, c, d ; 0,0)$.

configurations $C(\overline{\alpha a b})$ and $C(\overline{\gamma c d})$. In fact the value of a cell $c_{i, j}$ is unchanged, if and only if there hold the equations

$$
\bar{a}_{i}+\bar{b}_{j} \equiv c_{i}+d_{j} \quad(\bmod .2) .
$$

The condition given in (4.03) for all possible $m n$ combinations $i$ and $j$ gives us a division of the total $\Omega_{m, n}$ space into the sum of invariant area $I$ and alternating 


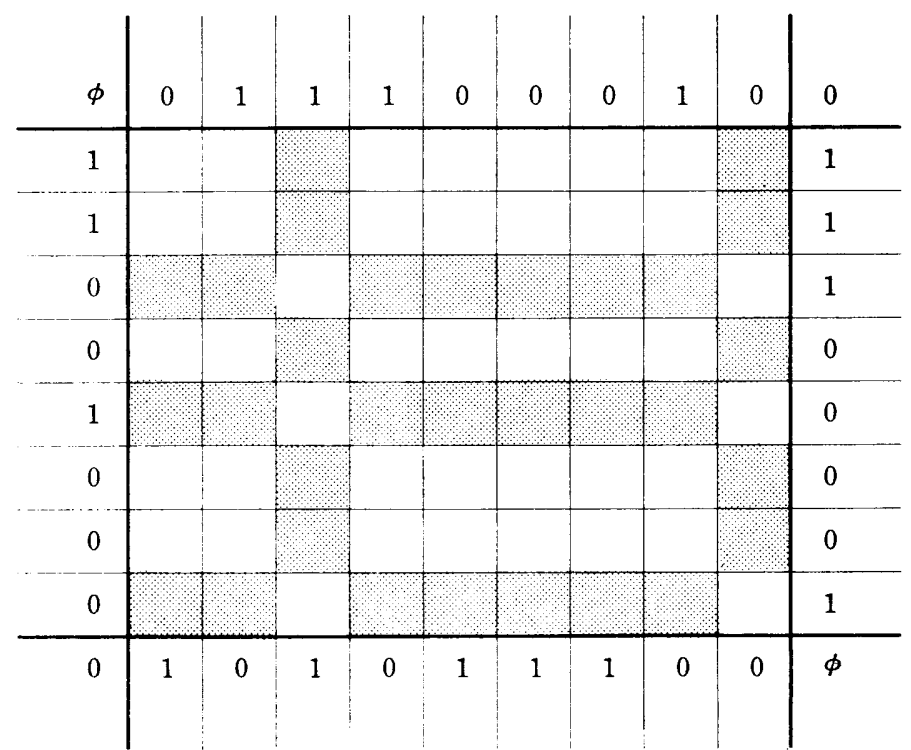

Fig. 4.2. I $\phi^{2} Q_{8,9}[a, b, c, d ; 1,0]$ shown by dotted domain.

area $A$, where $I$ consists of rectangular cell subspaces in which the value of each cell is constant, while in $A=\Omega_{m, n}-I$, each cell has the possibility of taking both of two values 1 and 0 . The situation is illustrated by Figure 4.2. More accurately

$$
\begin{aligned}
I & =I \phi^{2} \Omega_{m, n}[a, b, c, d ; 1,0] \\
A & =A \phi^{2} \Omega_{m, n}[a, b, c, d ; 1,0] \\
& =\phi^{2} \Omega_{n, n}[a, b, c, d ; 1,0]-I \phi^{2} \Omega_{m, n}[a, b, c, d ; 1,0] .
\end{aligned}
$$

We introduce

Definition 4.1. A cell $c_{i, j}$ in a cell space $\phi^{2} \Omega_{m, n}(C(X) ; a, b, c . d ; 1,0)$ is said to belong to the invariant class $I$ or to the alternating class $A$ respectively according to the situation

$$
a_{i}+b_{j} \equiv c_{i}+d_{j} \quad(\bmod .2)
$$

or

$$
a_{i}+b_{j} \equiv c_{i}+d_{j}+1 \quad(\bmod .2) .
$$

It is immediate to observe

LEMMA 4.1. In a cell space $\phi^{2} \Omega_{m, n}(C(X) ; a, b, c, d ; 1,0)$, there is a pair of partitions such that

$$
\begin{gathered}
m=m_{1}+m_{2}+\cdots+m_{k} \\
n=n_{1}+n_{2}+\cdots+n_{l}
\end{gathered}
$$

with positive integers $m_{i}$ and $n_{j}, 1 \leqq i \leqq k, 1 \leqq j \leqq l$, where $k$ and $l$ are subject to $1 \leqq$ $k \leqq m, 1 \leqq l \leqq n$. In correspondence with the pair of partitions given by (4.06) and (4.07) the whole $\Omega_{m, n}$ cell space is divided into $k$ subspaces, $\left\{S\left(m_{i, j}\right)\right\}(i=1,2, \cdots, k$; 
$j=1,2, \cdots, l)$ which are classified into two classes $I$ and $A$ given in Definition 4.1. The two classes occur alternatively in $\left\{S\left(m_{i}, n_{j}\right)\right\}$ (See Figure 4.4).

\begin{tabular}{c|cccccc|c}
$\dot{\phi}$ & $n_{1}$ & $n_{2}$ & $n_{3}$ & $n_{4}$ & $n_{5}$ & $n_{l}$ & $\gamma$ \\
\hline$m_{1}$ & $I$ & $A$ & $I$ & $A$ & $I$ & $A$ & \\
$m_{2}$ & $A$ & $I$ & $A$ & $I$ & $A$ & $I$ & \\
$m_{3}$ & $I$ & $A$ & $I$ & $A$ & $I$ & $A$ & \\
$m_{4}$ & $A$ & $I$ & $A$ & $I$ & $A$ & $I$ & \\
$m_{k}$ & $I$ & $A$ & $I$ & $A$ & $I$ & $A$ & \\
\hline$\alpha$ & & & & & & & $\phi$
\end{tabular}

Fig. 4.4.

Definition 4.2. The decomposition of $\Omega_{m, n}$ into a family of $\left\{S\left(m_{i}, n_{j}\right)\right\}(i=1,2$, $\cdots, k ; j=1,2, \cdots, l)$ is said to be an $I-A$ pattern inherited in the boundary configurations of $\phi^{2} \Omega_{m, n}(C(X) ; a, b, c, d ; 1,0)$.

With these preparations we can now reach the observations given by

THEOREM 4.1. In a cell space $\phi^{2} \Omega_{m, n}(C(X) ; a, b, c, d ; 1,0)$, the whole $\Omega_{m, n}$ space can be divided into the $I-A$ pattern inherited in the boundary configurations of $\phi^{2} \Omega_{m, n}(C(X) ; a, b, c, d ; 1,0)$. Any application of LMT in each subspace belonging to. the invariant class does invoke no change at all, while in each subspace belonging to. the alternating class it yields the change induced by the second term of the right-hand side of (4.01), which is discussed in Section 2.

\section{Summary and remarks}

We introduce a certain cell space $\phi^{2} \Omega_{m, n}(C(X) ; a, b, c, d ; \alpha, \gamma)$ with the invariant: boundary vectors $a, b, c, d$ and the invariant corners $\alpha, \gamma$ with two inhibition $\phi$. states in the opposite corners.

Starting in Section 2 with the particular case in which $a=(1,1, \cdots, 1)$, (m-dimension) $b=(1,1, \cdots, 1)$ (n-dimensional), $c=\bar{a}, d=\bar{b}, \alpha=1, \gamma=0$, in Section 3 we proceed to the so-called conjugate boundary configurations in which $c=\bar{a}, d=\bar{b}$, and finally in Section 4 we reach to the most general boundary configurations. What we establish in all of these Sections is to show that transitory aspects and final state aspects are common to those given in Section 2, where the notions of LLI sets and RUI sets play an important role.

\section{References}

[1] Kitagawa, T.: Oscillatory Phenomena in the Cell Space with inhibition states $\phi$, Information Science Approach to Biomathematics, X, Bull. Math. Statist., 15, No. 3-4 (1972) $1-9$.

[2] Kitagawa, T. and Yamaguchi, M.: Local Majority Transformations in Cell Space, Information Science Approach to Biomathematics, II, Bull. Math. Statist., 14, No. 3-4. (1971) 61-82. 